\title{
Comparison of family centered care with family integrated care and mobile technology (mFICare) on preterm infant and family outcomes: a multi-site quasi- experimental clinical trial protocol
}

Linda S. Franck ${ }^{1 *}\left(\mathbb{D}\right.$, Rebecca M. Kriz ${ }^{1}$, Robin Bisgaard ${ }^{2}$, Diana M. Cormier ${ }^{3}$, Priscilla Joe ${ }^{4}$, Pamela S. Miller ${ }^{5}$, Jae H. Kim ${ }^{6}$ Carol Lin ${ }^{7}$ and Yao Sun ${ }^{2}$

\begin{abstract}
Background: Family Centered Care (FCC) has been widely adopted as the framework for caring for infants in the Neonatal Intensive Care Unit (NICU) but it is not uniformly defined or practiced, making it difficult to determine impact. Previous studies have shown that implementing the Family Integrated Care (FICare) intervention program for preterm infants in the NICU setting leads to significant improvements in infant and family outcomes. Further research is warranted to determine feasibility, acceptability and differential impact of FICare in the US context. The addition of a mobile application (app) may be effective in providing supplemental support for parent participation in the FICare program and provide detailed data on program component uptake and outcomes.
\end{abstract}

Methods: This exploratory multi-site quasi-experimental study will compare usual FCC with mobile enhanced FICare (mFlCare) on growth and clinical outcomes of preterm infants born at or before 33 weeks gestational age, as well as the stress, competence and self-efficacy of their parents. The feasibility and acceptability of using mobile technology to gather data about parent involvement in the care of preterm infants receiving FCC or mFICare as well as of the mFICare intervention will be evaluated (Aim 1). The effect sizes for infant growth (primary outcome) and for secondary infant and parent outcomes at NICU discharge and three months after discharge will be estimated (Aim 2).

Discussion: This study will provide new data about the implementation of FICare in the US context within various hospital settings and identify important barriers, facilitators and key processes that may contribute to the effectiveness of FICare. It will also offer insights to clinicians on the feasibility of a new mobile application to support parent-focused research and promote integration of parents into the NICU care team in US hospital settings.

Trial registration: ClinicalTrials.gov, ID NCT03418870. Retrospectively registered on December 18, 2017.

Keywords: Family integrated care, Neonatal intensive care unit, Mobile application, Family centered care, Parents, Infants

\footnotetext{
* Correspondence: linda.franck@ucsf.edu

1 University of California San Francisco, School of Nursing, 2 Koret Way, Box

0606, San Francisco, CA 94143, USA

Full list of author information is available at the end of the article
}

(c) The Author(s). 2019 Open Access This article is distributed under the terms of the Creative Commons Attribution 4.0 International License (http://creativecommons.org/licenses/by/4.0/), which permits unrestricted use, distribution, and reproduction in any medium, provided you give appropriate credit to the original author(s) and the source, provide a link to the Creative Commons license, and indicate if changes were made. The Creative Commons Public Domain Dedication waiver (http://creativecommons.org/publicdomain/zero/1.0/) applies to the data made available in this article, unless otherwise stated. 


\section{Background}

Preterm birth is a leading cause of long-term disabilities and costs the US economy well over $\$ 26$ billion annually [1]. Poor growth during Neonatal Intensive Care Unit (NICU) hospitalization significantly increases a preterm infant's risk of mortality and serious long-term morbidity $[2,3]$. There is strong evidence that preterm infants who receive human milk have better growth and neurodevelopmental outcomes, reduced risk of major morbidities such as necrotizing enterocolitis (NEC), intraventricular hemorrhage (IVH), retinopathy of prematurity (ROP) and bronchopulmonary dysplasia (BPD), lower rates of nosocomial infection, and lower hospital costs [4-7]. Active parent involvement in preterm infant caregiving leads to higher breast feeding rates, earlier discharge and improved long-term neurodevelopment [8-12]. Secure parent-infant attachment and sensitivity to infant cues are essential to long-term quality of life for these infants and are significantly improved by NICU interventions that promote parenting self-efficacy and physical and emotional closeness between parents and infants [13-16]. Conversely, NICUrelated parent stress and depression adversely affect preterm children's long-term social, behavioral, and functional development $[17,18]$.

\section{Family-centered care (FCC)}

FCC is a philosophy and healthcare delivery framework that recognizes the central role of the family in an individual's health and well-being. Institutions and professionals that ascribe to FCC principles are expected to meaningfully engage patients and families in healthcare that is culturally and individually tailored and builds self-care skills, knowledge, confidence and shared decision-making [19-22]. FCC in NICUs is inconsistently conceptualized and practiced, resulting in confusion over the degree to which the care delivery models fully incorporate families as parterns in care [23]. Thus, the majority of patient care is delivered by NICU professionals, not parents [24-28]. Parent are still treated as "visitors", do not view themselves as their infant's primary caregiver, experience high levels of anxiety and stress, and often feel unprepared to care for their infant after discharge [29-35]. Geographic, racial/ethnic, and sociodemographic disparities in FCC have been reported by parents in the US with respect to the healthcare of their children and in attitudes of the multidisciplinary team [36-38].

\section{Family integrated care (FICare)}

Models of NICU care in which parents are the infant's primary caregivers have shown positive outcomes for infants and parents in both low and high-income settings [23, 39-41] but none have yet demonstrated sustainability or scalability. The Canadian FICare program is a promising new approach [42]. FICare differs from USpracticed FCC in the following ways:

1) Nurses receive formal education on how to teach parents to safely provide NICU care, and thus the focus shifts from the nurse to the parent as primary caregiver; 2) Parents spend a minimum of six hours per day in the NICU and attend group classes with a formal parentfocused curriculum on how to provide primary care of their infant; 3) Parents are explicitly incorporated into daily medical rounds, report on their infant and share in the clinical decisions; and 4) Trained "alumni" parents of former NICU infants provide support to the current NICU parents [42-45].

\section{Previous studies of FICare}

A 25-site cluster randomized trial (cRCT) across Canada $(n=18)$, Australia $(n=6)$ and New Zealand $(n=1)$ found that in NICUs randomized to FICare $(n=13 ; 827$ infants), infants had significantly improved 21-day weight gain (primary outcome) and a greater proportion of them received breast milk at discharge compared with usual care sites ( $n=12 ; 873$ infants) [46]. There was also lower maternal stress and anxiety in the FICare group. There were no significant between-group differences in the main neonatal morbidities (NEC, ROP, BPD). Analysis is ongoing for other outcomes. These results are compelling; however, FICare implementation required significant day-time involvement on the part of parents. The refusal rate based on time commitment required was approximately 43\%. Moreover, more Caucasian mothers and those working outside the home were enrolled in the FICare group, suggesting a selection bias perhaps linked to the time commitment required.

Results from a prospective parallel case-control study of FICare in China [47] showed significantly increased breastfeeding rates, breastfeeding duration, enteral nutrition duration, and weight gain at discharge [48], and higher scores on the mental development index and psychomotor development index at 18 months [49]. Of note, the FICare protocol in this study did not include peer-to-peer support from parents of former preterm infants and required parents to be in the hospital $3 \mathrm{~h}$ per day instead of $6 \mathrm{~h}$ per day. A FICare-based intervention bundle called Integrated Family Delivered Neonatal Care (IFDC) in the UK was shown to reduce overal length of stay and special care days and to shorten the time to full suck feeding compared with historical controls [50]. The IFDC bundle differed from FICare in that parents received additional support from IDFC coordinators and access to a free mobile app with educational information and a diary. Parents did not receive peer-to-peer support.

Additional studies are being conducted in Level II NICUs in Canada to evaluate length of stay, infant and 
maternal clinical outcomes, and cost [51] including breastfeeding self-efficacy and breastfeeding rates at discharge [52] with a lower acuity population.

\section{Knowledge gap}

There are many similarities, but also fundamental differences between US and Canadian health and social care systems that might impact the feasibility and outcomes of FICare. For example, US families do not have the same statutory paid parental leave benefits as Canadian parents [53] and have greater out of pocket expenses and healthcare administrative burden [54]. There is also evidence of differences in the composition of the NICU workforce, amount of NICU resources, and degree of parent participation in some types of decision-making between the countries $[55,56]$. Further innovation in the FICare program is clearly needed for the program to be feasibly and equitably implemented in US NICUs, particularly to improve accessibility of FICare for parents who live at a distance from the hospital, or have childcare or employment issues, and other significant social stressors. Another important knowledge gap is about the mechanism of action or relative efficacy of the FICare program components. The Canadian-led cRCT [46] was not designed for fine-grain analysis of the individual components of FICare to determine which were most strongly associated with the outcomes of interest overall or for specific subgroups. Further research will help better tailor the intervention to individual parent or infant characteristics, and to evaluate the post-discharge effects.

\section{Technology enhancement}

Mobile technology to engage and support US parents is rapidly advancing and may improve feasibility and accessibility of the FICare program. A systematic review of mobile health interventions for parents in neonatal intensive care units [57] found only eight studies, with the majority being of low or very low quality. The review found no clear impact on neonatal outcomes and no meta-analysis was conducted due to the heterogeneity of the studies. In addition to the IDFC app, we found one federally funded exploratory study of mobile technology to support parents of preterm infants, with that study focused solely on the transition from hospital to home [58]. Use of mobile technology with more interactive features in concert with FICare may enable greater parent participation in FICare when parents cannot be physically present in the NICU.

In partnership with parents and NICU healthcare professionals, we developed the We3health ${ }^{\mathrm{Tw}}$ app to serve as both a data collection platform for FCC and FICare research and to supplement the delivery of FICare program content for parents, especially for those who are unable to be present in the NICU during daytime hours. The present study will be the first known implementation and research of the Canadian FICare program in the US, with the added innovation of a mobile application, We3health ${ }^{\mathrm{Tw}}$, to facilitate parental education, clinical communication, parental support, and data collection.

\section{Methods and design \\ Aims}

The primary aim is to determine the feasibility and acceptability of mobile application technology to gather data about parent involvement in the care of NICU infants in the usual FCC and in the mFICare parent intervention.

The secondary aim is to compare usual FCC with mFICare on growth and clinical outcomes of preterm infants $\leq 33$ weeks gestational age, as well as the stress, competence and self-efficacy of their parents. The analysis will:

1 ) estimate the effect size for infant growth (primary outcome), defined as change in weight (z-score) at 21 days of age after enrollment, between the usual FCC and mFICare groups; and 2) estimate effect sizes for infant and parent outcomes (secondary outcomes). Infant clinical outcomes at NICU discharge include: weight gain velocity, rates of breastfeeding, nosocomial infection, necrotizing enterocolitis, intraventricular hemorrhage, retinopathy of prematurity, adverse events, and length of stay. Secondary parent outcomes include: perceived stress, parenting competence and parenting self-efficacy. Three-month post-discharge outcomes include: infant growth, breastfeeding, readmission rates and perceived parenting self-efficacy.

\section{Hypotheses}

The primary hypothesis is that the We3health ${ }^{\text {Tw }}$ mobile application will be feasible and acceptable, capturing high-quality data about the degree of parent involvement and the parent experience in FCC and mFICare conditions and enabling parents to access FICare content to supplement the in-person FICare interventions as well as extend access to FICare interventions for parents who cannot participate in some or all of the in-person FICare program. We secondarily hypothesize that participation in mFICare will be more effective than usual FCC in improving infant and parent outcomes at discharge and post-discharge within and across sites.

\section{Preliminary work}

We gathered extensive input from parents and front-line NICU healthcare professionals to inform the design of this research. We worked closely with parents of premature infants, the multidisciplinary team (nurses, physicians, respiratory therapists, social workers, psychologists, chaplains), and industry consultants in design thinking and mobile 
technology to adapt the FICare program to the US context. With support from the alumni parent-founded Will's Way Foundation and the UCSF Center for Digital Health Innovation, we developed the secure HIPAA-compliant We3health ${ }^{\text {tw }}$ mobile application to engage parents in key elements of the FICare program and to capture high quality data about parent involvement for all study participants with minimal burden. We have conducted preliminary user testing with NICU parents. All parents found the We3health ${ }^{\text {tx }}$ useful and gave positive feedback about the features. We engaged the multidisciplinary team in the NICU on how best to implement each component within their specific NICU context, including establishing nurse-led workgroups to facilitate implementation and address challenges throughout the study.

\section{Study design}

We will use an exploratory prospective sequential cohort quasi-experimental study design to assess the feasibility, acceptability and effect of mFICare in the NICU on infant and parent outcomes. Sequential enrollment will mitigate the between-group contamination or perceived inequities in quality of care that would likely occur with concurrent group enrollment at a single site. This design will resemble most closely the conditions of any single institution in a future cRCT. A parent/infant pair (primary pair) will be eligible for the study. Secondary pairs, for example a twin infant and the other parent from the same family, may be enrolled. Secondary pairs will not be included in the primary analysis but may be included in secondary analyses.

\section{Setting}

The study will be conducted in six NICUs throughout California. The multi-site design enables exploration of implementation barriers and facilitators and potential to scale the interventions across a variety of NICU types, including academic medical centers, freestanding children's hospitals, and community medical centers and with inborn and transported infants. The sites are also geographically and demographically diverse. A list of the participating sites can be found here [59].

\section{Participants and eligibility}

Parents (guardians) of infants born at 33 weeks gestation or less will be invited to participate in the trial. Participants will be excluded if: (1) the parent is not English literate, is less than 18 years of age, or does not have access to a hand-held computer (smart phone or tablet); or (2) the infant has a life-threatening congenital anomaly, is so critically ill that $s /$ he is unlikely to survive, or is receiving palliative care. Enrollment is limited to English literate parents because the We3health ${ }^{\text {tw }}$ version used for this study is only available in English.

\section{Recruitment}

Parent/infant primary pairs will be enrolled sequentially into one of two groups, usual FCC (Phase 1), then mFICare (Phase 2), at one of the six study sites. We aim to recruit 200 pairs for Phase 1 and 175 for Phase 2. The study has been approved by the institutional review boards at each site.

Parents of eligible infants will be given a study information sheet by clinical staff caring for the infants and, if interested in learning more, they will meet with a member of the research team to revew the informed consent information. This information will describe the study, including the different ways parents/guardians may participate, and the risks, benefits, and alternatives of each type of participation, and that they can withdraw from the study at any time without penalty to themselves or their infant's care. Those who agree will give their written informed consent to participate. Parents may request to defer approach or enrollment to a later date, as long as the infant is expected to remain in the NCU for a minimum of 21 days.

For multiple births, the primary infant will be selected by random assignment. Statistical staff from the university have prepared computer-generated random number schemes for twin and triplet births and placed the assignments in opaque envelopes numbered sequentially. Study site staff are blinded to the randomization schemes. Once a parent has signed the consent form, the study staff will retrieve the appropriate envelope in the sequence and open it to reveal which of the infants will be the primary infant for the study. If there are two parents/guardians who meet eligibility requirements, and both are eligible to participate, the parent who expects to spend the most time in the NICU will be designated as the "primary" pair for the primary analysis. Additional parent-infant pairs from the same family are nonindependent and therefore are excluded from the primary analyses. Once a parent has consented to be involved in the trial, they will be assigned a study ID number and contact information will be collected. The parent will be sent an invitation to download and register on We3health ${ }^{\text {tix }}$. Parents will receive a $\$ 25$ gift card at discharge, upon completion of all study questionnaires, and another $\$ 25$ gift card after completion of the 3month post-discharge questionnaire.

Nurses and physicians at the sites will be recruited to complete a questionnaires during the FCC and mFICare periods. They will receive an email explaining the project, risks, benefits and alternatives, and inviting them to complete the study questionnaires online. Their completion of the surveys indicates their consent to participate. Follow-up invitations will be sent to non-responders every two weeks until the questionnaires have been returned, or until a total of three reminder messages 
have been sent. Survey participants will be entered in a drawing to win one of four \$25 gift cards during each study phase.

\section{Intervention}

The usual FCC group will be recruited first. Data will be collected for this group without any change in the usual NICU practice. Recruitment for the mFICare group will begin after a break to allow any parents in the NICU who were in the FCC group to complete their study activities (about 2 months).

\section{Usual FCC group (Phase 1)}

Education Parents will receive an orientation to the unit by a NICU nurse and will have access to the usual written and video materials provided by the NICU. Parents will receive all NICU- required individualized parent teaching and support, delivered at the bedside by nurses, the Discharge Coordinator, other specialists, or in a discharge class.

Direct care Parents will be encouraged by nurses to participate in infant care under nursing supervision for feeding, bathing, dressing, and holding skin-to-skin. Social work and other support: Individualized support from social workers, developmental specialist, lactation consultants, physical therapy, occupational therapy, and other specialists will be offered.

Documentation Parents will not be asked to document any observations of their infant or their own skills acquisition. Nurses and Discharge Coordinators will complete the standard Discharge Teaching Checklist per usual hospital policies. Parents will be asked to use a version of the We3health ${ }^{\mathrm{Tn}}$ mobile application with limited features to track their time in the NICU, time learning and time spent in infant caregiving activities and to keep of a journal of their NICU experience. These data will not be shared with the clinical team.

\section{mFICare group (Phase 2)}

Clinical team and alumni parent training After completion of the usual FCC group enrollment, nurses who volunteer to provide care to mFICare infants/parents and the volunteer alumni parents will receive in-person and online training from the study team. The nurse training will follow the Canadian FICare curriculum. Alumni parent training will follow the Canadian FICare curriculum [43]. Physicians, therapists and social workers will receive in-person and online in-service education specific to their roles.
All elements of the FICare model are provided inperson. Additionally, education, daily medical rounds, peer support and documentation are supported by the We3health ${ }^{\text {Th }}$ app so that parents may access these components of the intervention at other times than offered in person or when they are away from the NICU.

Education Participants will be oriented to the unit by an mFICare nurse who will introduce them to the program, explain the parent's role as primary caregiver for their infant, and orient them to the NICU and parent resources, including We3health ${ }^{\mathrm{sx}}$. Parents will be provided the Parent Education program, a 3-week rotating curriculum offered a minimum of 3 afternoons per week $[43,46]$. The small group sessions will be facilitated by a member of the study team, clinical staff, or alumni parents. Parents will participate in-person or access the content remotely at a time of their choosing via We3health ${ }^{\mathrm{Tx}}$.

Direct care Parents will be treated as the infant's primary caregiver, with nurses serving as teachers and coaches. Parents will not be required to be in the NICU at specified hours as in the Canadian FICare program, but when in the NICU, they will be expected to provide as much infant care as they can, with support from mFICare-trained nurses. They will not provide ventilation management, intravenous fluid or intravenous medication administration.

Daily medical rounds Parents will be encouraged and supported to participate in daily medical rounds either in-person or remotely. Parents will report standardized data of their infant's status to the rounding care team ask questions, and reach consensus with the clinicians on the infant's daily plan. Nurses will provide rolemodeling and coaching to prepare parents for this role. Parents may begin by simply introducing themselves and their baby to the team and gradually increase their level of participation over time.

Peer Support Parents will be offered peer-to-peer support from alumni parents who will contact them remotely, by telephone, text or through We3health ${ }^{\mathrm{m}}$, at least twice weekly and as desired. Each site will build upon its existing peer support program or will be assisted by the study team to establish a new program. The sites will determine their selection criteria for alumni parents.

Documentation Parents will be expected to document time spent with their infant and record infant caregiving (eg skin-to-skin and feeding), observations and skills acquisition using We3health ${ }^{\text {Tw }}$. They will also use 
We3health ${ }^{\text {tw }}$ to keep a journal of observations of their infant and their own NICU experiences, which they can share with family, friends and the clinical team if they wish via email or social media links. Parents will access the mFICare skills checklist via We3health ${ }^{\mathrm{Tm}}$ and track their learning and skills acquisition. The infant observation data, parent-team communication and parent skills will be accessible to the clinical team and parents for rounds.

\section{Parent resources for both groups}

All parents, regardless of group assignment, will be provided with similar resources allowing for them to spend extended periods with their infant, facilitating breast feeding, and providing support for parents that may include reclining chairs, family lounges, kitchens, locked storage, wifi, and others. Resources may vary by site.

\section{Data collection}

\section{Clinical data}

Infant electronic medical record (EMR) data will be collected by study staff and include: demographics, antenatal/obstetric risks, delivery complications, admission illness severity scores, major clinical treatments (e.g., respiratory support, surgical procedures), adverse events, discharge diagnoses, disposition and length of stay.

\section{Parent survey data}

Parents will receive four sets of surveys - at enrollment (Baseline), 3 weeks after enrollment, at discharge, and at 3 months post-discharge (Table 1). Questionnaires for parents will be delivered via the We3health ${ }^{\mathrm{rm}}$. Baseline surveys will be delivered over 3 days to minimize survey burden. 3-week surveys will be delivered over 4 days. Paper questionnaires will be provided upon request. At 3 months post-discharge, parents will be sent an email with a link to secure online questionnaires or will be mailed questionnaires, depending on preference. Research assistants will be available to parents in both groups to support and encourage completion of the study surveys and We3health ${ }^{\text {Tix }}$ data.

\section{Outcome measures}

\section{Demographic data}

To assess the comparability of the study groups, demographic and medical information will be collected from the parent and infant medical record.

\section{Feasibility and acceptability of We3health ${ }^{\mathrm{TM}}$ and mFICare}

To measure the feasibility and acceptability of We3health ${ }^{\mathrm{m}}$, we will quantify the amount of parent usage of the application modules and their documentation of involvement with key care processes of infant caregiving, parenting skill acquisition and participation in the structured mFICare intervention (Phase 2 only). This will be compared with the level of involvement in these activities in the usual FCC condition. Specifically, we will use We3health ${ }^{\text {tw }}$ to measure attrition from the study; hours spent at infant's bedside; number of education sessions attended in-person, remotely or completed online; number of medical rounds sessions attended inperson or remotely; degree of active participation in medical rounds; amount of parent documentation completed; amount of participation in infant caregiving; skills acquired; and degree of independence in infant

Table 1 Parent measures

\begin{tabular}{|c|c|c|c|c|}
\hline Scale & Baseline & 21 days after enrollment & Discharge & Post-discharge \\
\hline Demographics $^{1}$ & $x$ & & & \\
\hline State \& Trait Anxiety [60] & $x$ & $x$ & $x$ & $x$ \\
\hline Parent Stressor Scale: NICU $[32,61]$ & $x$ & $x$ & & \\
\hline Family Centered Care [62] & $x$ & & $x$ & \\
\hline Perceived Parenting Self-Efficacy Tool (PMSE) [63] & & $x$ & $x$ & $x$ \\
\hline Nurse-Parent Support Tool [64] & & $x$ & & \\
\hline Readiness for Hospital Discharge Survey [65] & & & $x$ & \\
\hline mFICare Parent Survey (mFICare group only) & & & $x$ & \\
\hline Growth, Feeding current health ${ }^{1}$ & & & & $x$ \\
\hline Major life events ${ }^{1}$ & & & & $x$ \\
\hline What being the parent of a baby is like (WPL-R) [66] & & & & $x$ \\
\hline Duke Social Support [67] & & & & $x$ \\
\hline Edinburgh Postnatal Depression Scale [68] & & & & $x$ \\
\hline Life Orientation Test [69] & & & & $x$ \\
\hline Perinatal PTSD Questionnaire [70] & & & & $x$ \\
\hline
\end{tabular}

${ }^{1}$ Fixed choice and open-ended questions developed specifically for this study 
caregiving. To determine the acceptability of the mFICare intervention and of We3health ${ }^{\text {tw }}$ data collection method, questionnaires will be administered to parents at discharge and to the clinical team as described above.

\section{Primary outcome}

To measure the primary outcome of infant growth, weight gain by 21 days of age will be obtained from the infant medical record and z-scores will be calculated. The z-score refers to the exact number of standard deviations greater or smaller than the median and is the standard recommended metric for infant growth studies [71]. Change in weight from entry into the study to discharge, and weight gain velocity by 21 days postintervention will also be calculated to compare results with the FICare cRCT [46].

\section{Secondary outcomes}

Infant outcome measures are shown in Table 2. Secondary outcomes include: rate of human milk feeds at enrollment and discharge, time from birth to reaching full enteral feeds, length of NICU stay, and 5 major morbidities: (1) nosocomial infection [72]; (2) NEC [73]; (3) BPD [74]; (4) IVH [75]; and (5) ROP [76]. Number of adverse events for study infants and for the NICU per 1000 patient days for the usual FCC and mFICare enrollment periods will be compared. Parent stress will be assessed with multiple scales at different time points (see Table 1). Parents in the mFICare group will complete an additional FICare satisfaction questionnaire [25]. In the 3 -month post-discharge survey we will also measure 1) infant growth, measured as change in $\mathrm{z}$-scores and weight gain velocity from birth to 3 months after discharge; 2) proportion of infants receiving breastfeeding or human milk feeds; and 3) readmission rates.

\section{Clinical team outcomes}

Overall NICU nurse and physician attitudes and perceptions about FCC will be measured using the validated Family Centered Care Questionnaire [77] at two time- points: Prior to completion of the usual FCC enrollment, and prior completion of the mFICare enrollment. Nurses and physicians caring for mFICare infants will also complete the FICare satisfaction questionnaire.

Intervention fidelity checks will be done by study personnel during the clinical team and alumni parent training and periodically throughout the mFICare study period. We will conduct regular site visits that will involve auditing of study recruitment procedures, intervention implementation and data collection. Site research staff will also participate in monthly calls to discuss study progress, address any questions about study operating procedures, address any concerns about protocol implementation, and share best practices.

\section{Statistical considerations Sample size}

We expect 225 eligible NICU admissions at the first study site during the study period and anticipate enrolling 50 parent-infant pairs in each group (usual FCC and mFICare) at that site. Assuming 20\% attrition, we will have complete data on 40 infants per group. The study is exploratory at the other five sites to further assess feasibility and acceptability thus statistical power is based only on the first study site. The primary outcome, change in weight Z-score, typically has a standard deviation of between 0.44-0.47 (data from Toronto pilot and Canada-led cRCT). With a 0.05 null-hypothesis rejection threshold (alpha) for the two-tailed t-test, we will have $80 \%$ power to identify a group difference of $0.28-0.29$.

\section{Data analysis}

Descriptive statistics will be provided for all study variables: means and standard deviations for quantitative variables and frequencies and percentages for categorical variables. Data analysis will be based on an intention-totreat strategy with all participants enrolled in the usual FCC or mFICare groups analyzed within their respective groups regardless of compliance with the group assignment. Outcomes will be compared between the usual

Table 2 Infant measures

\begin{tabular}{|c|c|c|c|c|}
\hline & Baseline & 21 days after enrollment & Discharge & Post-discharge \\
\hline Change in infant weight (z-score) & & $x$ & & $X^{*}$ \\
\hline Amount of human milk/formula supplementation & $x$ & & $x$ & \\
\hline Frequency of breastfeeding & $x$ & & $X$ & $X^{*}$ \\
\hline Breastfeeding rate & $x$ & & $X$ & $X^{*}$ \\
\hline Length of stay & & & $x$ & \\
\hline Weight gain velocity & & & $x$ & \\
\hline $\begin{array}{l}\text { Major morbidities: Nosocomial infection, necrotizing enterocolitis, } \\
\text { intraventricular hemorrhage, retinopathy of prematurity, and adverse events. }\end{array}$ & & & $x$ & \\
\hline Hospital readmission rate & & & & $X^{*}$ \\
\hline
\end{tabular}


FCC and mFICare groups using t-tests for continuous variables, Wilcoxon tests for ordinal variables, and chisquare tests for categorical variables. Differences among the groups in potential confounding variables (e.g., site, GA, birth weight) will be examined. Variables that differ among the groups may be considered as covariates when examining group differences in the primary and secondary outcomes $[42,46]$. The covariate-adjusted scores may provide more precise estimates of the effect sizes. We will calculate confidence intervals around the differences because this will give a better idea of the range of values around the true means for the population. We will compare participation rates in specific aspects of usual FCC and mFICare such as caregiving activities, education, time spent with the infant or holding skin-toskin or the primary and secondary outcome variables to see if they differ for parents and infants with different characteristics (e.g., sex, race/ethnicity or distance between hospital and home). We will compare attitudes and perceived practices of nurses and physicians during the FCC and mFICare conditions.

\section{Confidentiality and data security}

Participants in the trial will be identified by a study ID only, with a master list linking names with numbers being held securely and separately from the study data. To ensure that all information is secure, data records will be kept in a secure location at each study site and on secure electronic systems (Salesforce ${ }^{\mathrm{m}}$ (San Francisco, CA); Qualtrics $^{\text {Tw }}$ (Provo, UT); REDCap ${ }^{\text {mix }}$ (Fort Lauderdale, FL)) and accessible only to research staff. As soon as all follow-up is completed the data records will be deidentified. De-identified data will be used for the statistical analysis and all publications will include only aggregated data.

The electronic version of the data will be maintained on secure servers protected by password. All hard copy patient identifiable data and electronic backup files will be kept in locked cabinets, which are held in a locked room accessed only by key and limited staff. Data files will be stored for ten years after completion of the project as recommended by the University of California Records Retention Schedule. Disposal of identifiable information will be shredded.

\section{Discussion}

High quality parent involvement in the care of NICU infants has longlasting positive effects on infant outcomes, particularly growth. However, the best methods for achieving this in the US context are not yet known. Results from this study will provide crucial data about the implementation of FICare in the US context within various hospital settings and identify important barriers, facilitators and key processes that may contribute to the effectiveness of FICare. It will also offer insights to clinicians on the feasibility of a new mobile application to support parent-focused research and greater accessibility to the FICare program to promote integration of parents into the NICU care team in US hospital settings.

Results from Phase 1 will provide useful data on current practices related to FCC, skin to skin, and breast feeding in the NICU setting. The Phase 1 results will also provide new information on the longitudinal experience of parents during and after their infant's NICU admission and potentially uncover disparities in experience based on parent or infant characteristics. Evaluation of parent collaboration in co-designing the We3health ${ }^{\text {Tm }}$ mobile application and the study will help to inform future collaborative research between researchers and communities. Results from Phase 2 will additionally provide useful data on the differential uptake and effects of the FICare components adapted to the US setting and the uptake and response of parents who utilize the FICare resources remotely through the We3health ${ }^{\mathrm{Tx}}$ mobile application. Finally, the tracking features of We3health ${ }^{\text {tw }}$ will enable enhanced data capture of parent engagement and involvement in infant caregiving. We will be able to track measures such as parent time spent in the NICU and in infant care activities such as skin-to-skin care or breastfeeding. This will allow us to measure key processes of engagement and parent outcome measures for all parents entered into the study (FCC and mFICare groups). These measures are otherwise extremely burdensome to collect and lead to poor quality data. We will also be able to examine parent use of educational resources and peer support, preferences for information and engagement strategies, and we will be able to ensure data quality. The findings from this study will be communicated to participants upon request, and to healthcare professionals and NICU parent advocacy groups through presentations and publications.

\section{Abbreviations \\ FCC: Family Centered Care; FICare: Family Integrated Care; mFICare: Mobile enhanced Family Integrated Care; NICU: Neonatal Intensive Care Unit}

\section{Acknowledgements \\ Not applicable.}

\section{Authors' contributions}

$L F, R B$, and $Y S$ were involved in the conception and design of the study. $L F$, $\mathrm{RK}, \mathrm{RB}$, and $\mathrm{YS}$ were all responsible for drafting of the initial protocol and were involved in the development of the study. LF, RK, RB, DC, PJ, PM, JK, CL, and YS were all involved in the implementation of the study and acquisition of data. All authors have had input into the protocol, read and given final approval of the final manuscript.

\section{Funding}

The study is supported as part of a larger gift to UCSF by Mark and Lynne Benioff through the UCSF California Preterm Birth Initiative. Contact: Dr. Larry Rand (Initiative PI), 3333 California St., Suite 285, San Francisco, CA 94118. The study funder has no role in any of the studiy activities. 


\section{Availability of data and materials}

Not applicable.

\section{Ethics approval and consent to participate}

The study has been approved by the following ethics committees: University of California, San Francisco (16-19542), Benioff Children's Hospital Oakland (2017-013), Community Regional Medical Center (2017096), and Kaiser Permanente (00001045). All participants will give written informed consent to participate in this study.

\section{Consent for publication}

Not applicable.

\section{Competing interests}

The authors declare that they have no competing interests.

\section{Author details}

'University of California San Francisco, School of Nursing, 2 Koret Way, Box 0606, San Francisco, CA 94143, USA. ${ }^{2}$ UCSF Benioff Children's Hospital, San Francisco, 1975 4th St, San Francisco, CA 94158, USA. ${ }^{3}$ Community Regional Medical Center, 2823 Fresno St, Fresno, CA 93721, USA. ${ }^{4}$ Division of Neonatology, UCSF Benioff Children's Hospital, Oakland, 747 52nd Street, Oakland, CA 94618, USA. ${ }^{5}$ UCLA Health, 924 Westwood Boulevard, Suite 720, Los Angeles, CA 90024, USA. ${ }^{6}$ Division of Neonatology, Perinatal Institute, Cincinnati Children's Hospital Medical Center, 3333 Burnet Avenue, MLC 7009, Cincinnati, OH 45229, USA. ${ }^{7}$ Kaiser Permanente Santa Clara, 3rd Floor Dept. 340.710 Lawrence Expy, Santa Clara, CA 95051, USA.

Received: 1 July 2019 Accepted: 15 November 2019 Published online: 02 December 2019

\section{References}

1. Institute of Medicine (US) Committee on Understanding Premature Birth and Assuring Healthy Outcomes; Behrman RE, Butler AS, editors. Preterm Birth: Causes, Consequences, and Prevention. Washington (DC): National Academies Press (US); 2007.

2. Franz AR, Pohlandt F, Bode H, Mihatsch WA, Sander S, Kron M, et al. Intrauterine, early neonatal, and postdischarge growth and neurodevelopmental outcome at 5.4 years in extremely preterm infants after intensive neonatal nutritional support. Pediatr. 2009;123(1):e101-9.

3. Belfort MB, Rifas-Shiman SL, Sullivan T, Collins CT, McPhee AJ, Ryan P, et al. Infant growth before and after term: effects on neurodevelopment in preterm infants. Pediatr. 2011;128(4):e899-906.

4. Cristofalo EA, Schanler RJ, Blanco CL, Sullivan S, Trawoeger R, KiechlKohlendorfer $\mathrm{U}$, et al. Randomized trial of exclusive human milk versus preterm formula diets in extremely premature infants. J Pediatr. 2013;163(6): 1592-1595.e1.

5. Porcelli PJ, Weaver RG. The influence of early postnatal nutrition on retinopathy of prematurity in extremely low birth weight infants. Early Human Dev. 2010;86(6):391-6.

6. Johnson TJ, Patel AL, Bigger HR, Engstrom JL, Meier PP. Economic benefits and costs of human Milk feedings: a strategy to reduce the risk of prematurity-related morbidities in very-low-birth-weight infants. Adv Nutr. 2014;5(2):207-12.

7. Chowning R, Radmacher P, Lewis S, Serke L, Pettit N, Adamkin DH. A retrospective analysis of the effect of human milk on prevention of necrotizing enterocolitis and postnatal growth. J Perinatol. 2016;36(3):221-4.

8. Flacking R, Ewald U, Wallin L. Positive effect of kangaroo mother care on long-term breastfeeding in very preterm infants. J Obstet Gynecol Neonatal Nurs. 2011;40(2):190-7.

9. Reynolds LC, Duncan MM, Smith GC, Mathur A, Neil J, Inder T, et al. Parental presence and holding in the neonatal intensive care unit and associations with early neurobehavior. J Perinatol. 2013;33(8):636-41.

10. Feldman R, Rosenthal Z, Eidelman Al. Maternal-preterm skin-to-skin contact enhances child physiologic organization and cognitive control across the first 10 years of life. Biol Psychiatry. 2014;75(1):56-64.

11. Zhang X, Kurtz M, Lee S-Y, Liu H. Early intervention for preterm infants and their mothers: a systematic review. J Perinat Neonatal Nurs. 2014;18.

12. Casavant SG, McGrath JM, Burke G, Briere C-E. Caregiving factors affecting breastfeeding duration within a neonatal intensive care unit. Adv Neonatal Care. 2015;15(6):421-8.
13. Newnham CA, Milgrom J, Skouteris H. Effectiveness of a modified motherinfant transaction program on outcomes for preterm infants from 3 to 24 months of age. Infant Behav Dev. 2009;32(1):17-26.

14. Forcada-Guex M, Pierrehumbert B, Borghini A, Moessinger A, Muller-Nix C. Early dyadic patterns of mother-infant interactions and outcomes of prematurity at 18 months. Pediatr. 2006;118(1):e107-14.

15. Hane AA, Myers MM, Hofer MA, Ludwig RJ, Halperin MS, Austin J, et al. Family nurture intervention improves the quality of maternal caregiving in the neonatal intensive care unit: evidence from a randomized controlled trial. J Dev Behav Pediatr. 2015;36(3):188-96.

16. Welch MG, Firestein MR, Austin J, Hane AA, Stark RI, Hofer MA, et al. Family nurture intervention in the neonatal intensive care unit improves socialrelatedness, attention, and neurodevelopment of preterm infants at 18 months in a randomized controlled trial. J Child Psychol Psychiatry. 2015; 56(11):1202-11.

17. McManus BM, Poehlmann J. Maternal depression and perceived social support as predictors of cognitive function trajectories during the first 3 years of life for preterm infants in Wisconsin. Child Care Health Dev. 2012; 38(3):425-34.

18. Rahkonen P, Heinonen K, Pesonen A-K, Lano A, Autti T, Puosi R, et al. Mother-child interaction is associated with neurocognitive outcome in extremely low gestational age children. Scand J Psychol. 2014;55(4):311-8.

19. Kuo DZ, Houtrow AJ, Arango P, Kuhlthau KA, Simmons JM, Neff JM. Familycentered care: current applications and future directions in pediatric health care. Matern Child Health J. 2012;16(2):297-305.

20. Kitson A, Marshall A, Bassett K, Zeitz K. What are the core elements of patient-centred care? A narrative review and synthesis of the literature from health policy, medicine and nursing. J Adv Nurs. 2013;69(1):4-15.

21. Institute of Medicine. Partnering with Patients to Drive Shared Decisions, Better Value, and Care Improvement: Workshop Proceedings. DC: The National Academies Press; 2014.

22. Johnson BH. Family-centered care: four decades of progress. Fam Syst Health. 2000;18(2):137-56.

23. Franck LS, O'Brien K. The evolution of family-centered care: from supporting parent-delivered interventions to a model of family integrated care. Birth Defects Res. 2019:111(15):1044-59.

24. Dunn MS, Reilly MC, Johnston AM, Hoopes RD, Abraham MR. Development and Dissemination of Potentially Better Practices for the Provision of FamilyCentered Care in Neonatology: The Family-Centered Care Map. Pediatr. 2006;118(Supplement 2):S95-107.

25. Gooding JS, Cooper LG, Blaine Al, Franck LS, Howse JL, Berns SD. Family support and family-centered Care in the Neonatal Intensive Care Unit: origins, advances. Impact Sem Perinatol. 2011;35(1):20-8.

26. Petersen MF, Cohen J, Parsons V. Family-centered care: do we practice what we preach? JOGNN. 2004;33(4):421-7.

27. Berns SD, Boyle MD, Popper B, Gooding JS, Preemie Health Coalition. Results of the premature birth national need-gap study. J Perinatol. 2007; 27(Suppl 2):S38-44.

28. Cooper L, Paap K, Alter C, Manweiler J, Russell R. Peering in: a National Survey of family-centered policies and practices in the NICU. Poster presented at: Care of the High Risk Newborn Conference; 2013; Clearwater, Florida.

29. Fenwick J, Barclay L, Schmied V. Craving closeness: a grounded theory analysis of women's experiences of mothering in the special care nursery. Women Birth. 2008;21(2):71-85.

30. Fenwick J, Barclay L, Schmied V. Struggling to mother: a consequence of inhibitive nursing interactions in the neonatal nursery. The J Perinat Neonat Nurs. 2001;15(2):49-64.

31. Alves E, Rodrigues C, Fraga S, Barros H, Silva S, Alves E. Parents' views on factors that help or hinder breastmilk supply in neonatal care units: systematic review. Arch Dis Child Fetal Neonatal Ed. 2013;98(6):F511-7.

32. Franck $L S, \operatorname{Cox} \mathrm{S}$, Allen $\mathrm{A}$, Winter I. Measuring neonatal intensive care unitrelated parental stress. J Adv Nurs. 2005;49(6):608-15

33. Murdoch MR, Franck LS. Gaining confidence and perspective: a phenomenological study of mothers' lived experiences caring for infants at home after neonatal unit discharge. J Adv Nurs. 2012;68(9):2008-20.

34. Lyndon A, Wisner K, Holschuh C, Fagan KM, Franck LS. Parents' perspectives on navigating the work of speaking up in the NICU. JOGNN. 2017;46(5):716-26.

35. Garfield CF, Lee Y, Kim HN. Paternal and maternal concerns for their very low-birth-weight infants transitioning from the NICU to home. J Perinat Neonatal Nurs. 2014;28(4):305-12. 
36. Azuine RE, Singh GK, Ghandour RM, Kogan MD. Geographic, Racial/Ethnic, and Sociodemographic Disparities in Parent-Reported Receipt of FamilyCentered Care among US Children [Internet]. Int J Family Med. 2015 [cited 2018. Available from: https://www.hindawi.com/journals/ijfm/2015/168521/

37. Lake ET, Staiger D, Horbar J, Kenny MJ, Patrick T, Rogowski JA. Disparities in perinatal quality outcomes for very low birth weight infants in neonatal intensive care. Health Serv Res. 2015;50(2):374-97.

38. Hendson L, Reis MD, Nicholas DB. Health care providers' perspectives of providing culturally competent care in the NICU. JOGNN. 2015;44(1):17-27.

39. Levin A. The mother-infant unit at Tallinn Children's hospital, Estonia: a truly baby-friendly unit. Birth. 1994;21(1):39-44 discussion 45-46.

40. Bhutta ZA, Khan I, Salat S, Raza F, Ara H. Reducing length of stay in hospital for very low birthweight infants by involving mothers in a stepdown unit: an experience from Karachi (Pakistan). BMJ. 2004;329(7475):1151-5.

41. Ortenstrand A, Westrup B, Broström EB, Sarman I, Akerström S, Brune T, et al. The Stockholm neonatal family centered care study: effects on length of stay and infant morbidity. Pediatr. 2010;125(2):e278-85.

42. O'Brien K, Bracht M, Macdonell K, McBride T, Robson K, O'Leary L, et al. A pilot cohort analytic study of family integrated Care in a Canadian neonatal intensive care unit. BMC Pregnancy Childbirth. 2013;13(1):S12.

43. Macdonell K, Christie K, Robson K, Pytlik K, Lee SK, O'Brien K. Implementing family-integrated care in the NICU: engaging veteran parents in program design and delivery. Adv Neonatal Care. 2013;13(4):262-9 quiz 270-1.

44. Jiang S, Warre R, Qiu X, O'Brien K, Lee SK. Parents as practitioners in preterm care. Early Human Dev. 2014;90(11):781-5.

45. Bracht M, O'Leary L, Lee SK, O'Brien K. Implementing family-integrated care in the NICU: a parent education and support program. Adv Neonatal Care. 2013;13(2):115-26.

46. O'Brien K, Robson K, Bracht M, Cruz M, Lui K, Alvaro R, da Silva O, Monterrosa L, Narvey M, Ng E, Soraisham A, Ye XY, Mirea L, Tarnow-Mordi W, Lee SK. FICare study group and FICare parent advisory board. Effectiveness of family integrated care in neonatal intensive care units on infant and parent outcomes: a multicentre, multinational, clusterrandomised controlled trial. Lancet Child Adolesc Health. 2018;2(4):245-54

47. Hei M, Gao X, Gao X, Nong S, Zhang A, Zhang Q, et al. Is family integrated care in neonatal intensive care units feasible and good for preterm infants in China: study protocol for a cluster randomized controlled trial. Trials. 2016;17:22.

48. He S-W, Xiong Y-E, Zhu L-H, Lv B, Gao X-R, Xiong H, et al. Impact of family integrated care on infants' clinical outcomes in two children's hospitals in China: a pre-post intervention study. Ital J Pediatr. 2018;44(1):65.

49. Li Y, Gao XY, Xiang XY, Dai HM, Yang L, Shoo KMY, et al. Effect of family integrate care on the development of preterm infants at 18 months of age. Zhonghua Er Ke Za Zhi. 2016;54(12):902-7.

50. Banerjee J, Aloysius A, Mitchell K, Silva I, Rallis D, Godambe SV, et al Improving infant outcomes through implementation of a family integrated care bundle including a parent supporting mobile application. Arch Dis Child Fetal Neonatal Ed. 2019; fetalneonatal-2018-316435.

51. Benzies KM, Shah V, Aziz K, Isaranuwatchai W, Palacio-Derflingher L, Scotland J, et al Family Integrated Care (FICare) in Level II Neonatal Intensive Care Units: study protocol for a cluster randomized controlled trial. Trials [Internet]. 201710 [cited 2018 Nov 20];18. Available from: https:// www.ncbi.n/m.nih.gov/pmc/articles/PMC5634877/

52. Brockway M, Benzies KM, Carr E, Aziz K. Breastfeeding self-efficacy and breastmilk feeding for moderate and late preterm infants in the family integrated care trial: a mixed methods protocol. Int Breastfeed J. 2018;13:29.

53. Waldfogel J. International policies toward parental leave and child care. Future Child. 2001 Spring-Summer;11(1):98-111.

54. LaPierre TA. Comparing the Canadian and US systems of health care in an era of health care reform. J Health Care Finance. 2012;38(4):1-18.

55. Thompson LA, Goodman DC, Little GA. Is more neonatal intensive care always better? Insights from a cross-national comparison of reproductive care. Pediatr. 2002;109(6):1036-43.

56. Verhagen AAE, Janvier A, Leuthner SR, Andrews B, Lagatta J, Bos AF, et al. Categorizing neonatal deaths: a cross-cultural study in the United States, Canada, and the Netherlands. J Pediatr. 2010;156(1):33-7.

57. Dol J, Delahunty-Pike A, Anwar Siani S, Campbell-Yeo M. eHealth interventions for parents in neonatal intensive care units: a systematic review. JBI Database Syst Rev Implement Rep. 2017;15(12):2981-3005.

58. Agency for Healthcare Reserach and Quality. NICU-2-HOME: Using Health IT to Support Parents of NICU Graduates Transitioning to Home (Illinois) AHRQ National Resource Center; Health Information Technology: Best
Practices Transforming Quality, Safety, and Efficiency [Internet]. [cited 2018 Oct 17]. Available from: https:/healthit.ahrq.gov/ahrq-funded-projects/ nicu-2-home-using-health-it-support-parents-nicu-graduates-transitioninghome

59. California Preterm Birth Initiative. Interventions Across the Reproductive Lifecourse: Postnatal Care [Internet]. [cited 2019 Jan 15]. Available from: https://pretermbirthca.ucsf.edu/interventions-across-reproductive-lifecoursepostnatal-care

60. Spielberger CD, Gorsuch RL, Lushene RE. Manual for the state-trait anxiety inventory. Palo Alto, CA: Consulting Psychologists Press; 1970.

61. Miles MS, Funk SG, Kasper MA. The neonatal intensive care unit environment: sources of stress for parents. AACN Clin Issues Crit Care Nurs. 1991;2(2):346-54.

62. Raiskila S, Lehtonen L, Tandberg BS, Normann E, Ewald U, Caballero S, et al. Parent and nurse perceptions on the quality of family-centred care in 11 European NICUs. Australian Critical Care. 2016;29(4):201-9.

63. Barnes CR, Adamson-Macedo EN. Perceived maternal parenting self-efficacy (PMP S-E) tool: development and validation with mothers of hospitalized preterm neonates. J Adv Nurs. 2007;60(5):550-60.

64. Miles MS, Carlson J, Brunssen S. The nurse parent support tool. J Pediatr Nurs. 1999;14(1):44-50.

65. Weiss M, Johnson NL, Malin S, Jerofke T, Lang C, Sherburne E. Readiness for discharge in parents of hospitalized children. J Pediatr Nurs. 2008;23(4):282-95.

66. Pridham KF, Chang AS. What being the parent of a new baby is like: revision of an instrument. Res Nurs Health. 1989;12(5):323-9.

67. Broadhead WE, Gehlbach SH, de Gruy FV, Kaplan BH. The Duke-UNC functional social support questionnaire. Measurement of social support in family medicine patients. Med Care. 1988;26(7):709-23.

68. Cox JL, Holden JM, Sagovsky R. Detection of postnatal depression. Development of the 10-item Edinburgh postnatal depression scale. $\mathrm{Br}$ J Psychiatry. 1987;150:782-6.

69. Scheier MF, Carver CS, Bridges MW. Distinguishing optimism from neuroticism (and trait anxiety, self-mastery, and self-esteem): a reevaluation of the life orientation test. J Pers Soc Psychol. 1994;67(6):1063-78.

70. Callahan $J \mathrm{~L}$, Borja SE. Psychological outcomes and measurement of maternal posttraumatic stress disorder during the perinatal period. J Perinat Neonatal Nurs. 2008;22(1):49-59.

71. Fenton TR, Sauve RS. Using the LMS method to calculate $z$-scores for the Fenton preterm infant growth chart. Eur J Clin Nutr. 2007;61(12):1380-5.

72. Garner JS, Jarvis WR, Emori TG, Horan TC, Hughes JM. CDC definitions for nosocomial infections, 1988. Am J Infect Control. 1988;16(3):128-40.

73. Walsh MC, Kliegman RM. Necrotizing Enterocolitis: treatment based on staging criteria. Pediatr Clinics North America. 1986;33(1):179-201.

74. Shennan AT, Dunn MS, Ohlsson A, Lennox K, Hoskins EM. Abnorma pulmonary outcomes in premature infants: prediction from oxygen requirement in the neonatal period. Pediatr. 1988;82(4):527-32.

75. Canadian Pediatric Society Statement. Routine screening cranial ultrasound examinations for the prediction of long term neurodevelopmental outcomes in preterm infants. Paediatr Child Health. 2001;6(1):39-43.

76. International Committee for the Classification of Retinopathy of Prematurity. The international classification of retinopathy of prematurity revisited. Arch Ophthalmol. 2005;123(7):991-9.

77. Bruce B, Letourneau N, Ritchie J, Larocque S, Dennis C, Elliott MR. A multisite study of health professionals' perceptions and practices of familycentered care. J Fam Nurs. 2002;8(4):408-29.

\section{Publisher's Note}

Springer Nature remains neutral with regard to jurisdictional claims in published maps and institutional affiliations. 